Pesq. Vet. Bras. 36(5):453-459, maio 2016 DOI: $10.1590 / \mathrm{S} 0100-736 \mathrm{X} 2016000500016$

\title{
Influência da suplementação de vitaminas A, D e E na função imune de bezerros alimentados com dieta á base de feno capim tifton (Cynodon spp.) ${ }^{1}$
}

\author{
Endrew R.M. Martins ${ }^{2}$, Heloisa G. Bertagnon ${ }^{2,3 *}$, Camila F. Batista ${ }^{2}$, Renata C. \\ Gomes $^{2}$, Kamila R. Santos ${ }^{2}$, Jéssyca B. Bellinazzi² e Alice M.M.P. Della Libera ${ }^{2}$
}

\begin{abstract}
Martins E.R.M., Bertagnon H.G., Batista C.F., Gomes R.C., Santos K.R., Bellinazzi J.B. \& Della Libera A.M.M.P. 2016. [Influence of A, D and E vitamin supplements on immune function of calves fed diet tifton (Cynodon spp.) hay.] Influência da suplementação de vitaminas $\mathrm{A}, \mathrm{D}$ e $\mathrm{E}$ na função imune de bezerros alimentados com dieta à base de feno de capim-tifton (Cynodon spp.). Pesquisa Veterinária Brasileira 36(5):453-459. Faculdade de Medicina Veterinária e Zootecnia, Universidade de São Paulo, Av. Prof. Dr. Orlando Marques de Paiva 87, Cidade Universitária, São Paulo, SP 05508-270, Brazil. E-mail: hgodoi@usp.br

Vitamins are organic compounds which are required in small quantities in the body, however essential for the metabolic functions. They participate in numerous metabolic reactions, physiological and immune cells, needed to maintain animal health, as well as act as immunostimulants. Although the diet rich in fresh green foliage provides sufficient amounts of vitamin A, D and E, intensive supplementation with food stored in the form of hay or silage can reduce up to $50 \%$ of the levels of these vitamins in food. Given this, the proposal of this study was to verify how the parenteral administration of vitamins ADE acts as immunostimulant in steers fed exclusively with hay of tifton. For that, 14 cattle were divided into two homogeneous groups: Group S, supplemented with vitamin A, D e E given in a single intramuscular dose of $30 \mathrm{~mL}$, and Group C without supplementation. Both groups were housed in private stalls and fed with hay for a period of three months. Immune evaluation was performed by blood count and testing of leukocyte function (oxidative metabolism and phagocytosis) in the moments before treatment, three and ten days after the treatments. Considering that supplementation with vitamin A, D e E increased the percentage of granulocytic cell activity and the intensity of the activity of mononuclear cells, as well as intensified the antioxidant effect prolonging the survival of red blood cells and neutrophils, it can be concluded that this treatment had a beneficial effect on the immune response of Holstein calves, despite the damaging effects of exclusive feeding hay, and the partially deprivation of solar incidence.
\end{abstract}

INDEX TERMS: Vitamin A, D and E, immune function, calves, tifton, Cynodon spp., hay, cattle, phagocytosis, ROS, hemogram.

RESUMO.- As vitaminas são compostos orgânicos necessários em poucas quantidades no organismo, todavia indispensáveis para as funções metabólicas. Elas se inserem em

\footnotetext{
${ }^{1}$ Recebido em 21 de outubro de 2015.

Aceito para publicação em 15 de março de 2016.

${ }^{2}$ Faculdade de Medicina Veterinária e Zootecnia (FMVZ), Universidade de São Paulo (USP), Av. Prof. Dr. Orlando Marques de Paiva, 87, Cidade Universitária, São Paulo, SP 05508-270, Brasil. *Autor para correspondência: hgodoi@usp.br

${ }^{3}$ Departamento de Medicina Veterinária, Universidade do Centro Oeste do Paraná (UNICENTRO), Rua Simeão Camargo Varela de Sá, n.03, CEDETEG, Guarapuava, PR 85040-080, Brasil.
}

inúmeras reações metabólicas, fisiológicas e imunes das células, necessárias para a manutenção da saúde animal, além de atuarem como imunoestimulante. Embora a dieta rica em folhagens verdes frescas forneça quantidades suficientes de vitaminas A, D e E a suplementação intensiva com alimentos conservados na forma de feno ou silagem pode reduzir em até $50 \%$ dos teores destas vitaminas no alimento. Diante disso, a proposta do trabalho foi verificar se a administração parenteral de vitaminas A, D e E age como imunoestimulante em garrotes estabulados e alimentados exclusivamente com feno de tifton. Para tanto 14 bovinos foram divididos em dois grupos homogêneos, sendo 
o grupo $S$, suplementado com vitamina A, D e E em dose única de $30 \mathrm{~mL}$ por via intramuscular; e o grupo $C$, sem suplementação. Ambos os grupos foram alojados em baias parcialmente privadas de sol, e alimentados com feno por um período de três meses. A avaliação imune foi realizada por hemogramas e ensaio de função leucocitária (metabolismo oxidativo e fagocitose) nos momentos antes do tratamento, três e dez dias após os tratamentos. Tendo em vista que a suplementação com polivitamínicos A, D e E aumentou a porcentagem da atividade de células granulocítica e a intensidade da atividade de células mononucleares, além de intensificar o efeito antioxidante prolongando a sobrevida de hemácias e neutrófilos, conclui-se que esta suplementação promoveu efeito benéfico na resposta imune de bezerros da Raça Holandesa, apesar dos efeitos deletérios da alimentação exclusiva com feno e da privação parcial da incidência solar direta.

TERMOS DE INDEXAÇÃO: Vitaminas A, D e E, função imune, bezerros, capim-tifton, Cynodon spp., feno, bovino, fagocitose, ERO, hemograma.

\section{INTRODUÇÃO}

O uso de suplementação com vitaminas para bovinos, especialmente $\mathrm{A}, \mathrm{D}$ e E, é uma prática comum na pecuária, seja no intuito de aumentar a produtividade (Carnagey et al. 2008, Baldin et al. 2013), prevenir incidência de doenças (Ferreira et al. 2007) ou como adjuvante no tratamento de doenças infecciosas (Mukherjee 2008, Lopes et al. 2009).

Estes efeitos benéficos estão correlacionados com a maior efetividade do sistema imune, observado por menor estresse oxidativo e maior atividade fagocítica e bactericida dos fagócitos bovinos (Paschoal, Zanetti \& Cunha 2003, Bouwstra et al. 2008, Urban-Chmiel et al. 2009), devido a melhorias na sinalização intracelular de neutrófilos e maiorrprodução de espécies reativas de oxigênio (ERO), que embora seja benéfica para a eliminaçãode patógenos fagocitados, destroe as células hospedeiras quando o sistema antioxidante não é efetivo (Higuchi \& Nagahata 2000). Esta lipoperoxidação de membranas de células sadias resulta em necrose celular e consequentemente progressão da inflamação via liberação de citocinas inflamatórias intracelulares (Riegel 2002, Lambeth 2004, Carnagey et al. 2008).

Apesar das vitaminas funcionarem como imunoestimulantes, alguns autores não encontraram vantagens na suplementação destas vitaminas em bovinos, sugerindo que a própria alimentação, quando composta por forragens frescas e exposição ao sol já seriam suficientes para o animal atingir o requerimento destes nutrientes (Ferreira et al. 2007, Politis 2012). No entanto, em períodos de mudanças climáticas a oferta de forragem diminui, bem como o valor nutricional das pastagens, sendo muito comum, a suplementação intensiva com alimentos conservados na forma de feno ou silagem, processo que pode reduzir em até $50 \%$ os teores de vitamina A e E no alimento (McDowell et al. 1996). Além disto, animais estabulados sofrem privação de luz solar, reduzindo a síntese de vitamina D no organismo (McDowell et al. 1996).

Pouco se sabe sobre a influência do efeito da suplementação frente a uma dieta potencialmente deficiente nestas vitaminas como único desafio imunológico. A maioria das pesquisas conduziram seus estudos em animais submetidos a desafios imunológicos complexos como o transporte (Urban-Chmiel et al. 2009), o inicio da lactação (Calderón et al. 2007, Ferreira et al. 2007, Bouwstra et al. 2008, Graugnard et al. (2012) ou em animais doentes (Mukherjee 2008, Lopes et al. 2009), não ficando claro se a suplementação vitamínica apresentaria efeito em desafios mais tênues.

Assim, considerando que animais estabulados e alimentados somente com feno de tifton podem estar com déficit destas três vitaminas, este trabalho tem o intuito de verificar se a administração parenteral de vitaminas ADE pode agir como imunoestimulante em bovinos jovens sadios.

\section{MATERIAL E MÉTODOS}

O delineamento utilizado foi o inteiramente casualizado, no qual 14 bovinos sadios da raça holandesa, machos, com idade de 10 meses ( \pm 2 meses) e peso de $300 \mathrm{~kg}( \pm 25 \mathrm{~kg})$, foram alojados em baias com privação parcial de incidência solar, com cama de maravalha, acesso a água ad libitum e alimentados exclusivamente com feno de tifton duas vezes ao dia. Todos os animais nasceram a termo e mamaram colostro. Foram encaminhados a Clínica de Bovinos e Pequenos Ruminantes da Faculdade de Medicina Veterinária e Zootecnia, Universidade de São Paulo (FMVZ-USP) aos 15 dias de vida e permaneceram neste setor até o inicio do experimento. Foram desmamados aos 60 dias de vida pesando aproximadamente $60 \mathrm{~kg}( \pm 5 \mathrm{~kg}$ ) e vermifugados (albendazole via oral na dose de $10 \mathrm{mg} / \mathrm{kg}$ ) aos 2 e aos 8 meses de idade. Semanalmente foram submetidos a exames físicos e hemograma como critério de sanidade, não evidenciando alterações nestes exames. A partir dos 7 meses de idade o concentrado (novilhina- Purina ${ }^{\circledR}$ - $2 \mathrm{~kg}$ / animal/dia) foi removido da alimentação e manteve-se a dieta exclusivamente com feno de Capim-Tifton (Cynodon spp.). Aos 10 meses de idade, os bovinos foram distribuidos em dois grupos homogêneos (com sete animais cada), sendo o grupo controle (C) composto por animais que não receberam nenhum tipo de suplementação e o grupo suplementado (S), por animais que foram tratados com vitaminas A, D e E (Adevita, Vétoquinol ${ }^{\circledR}$, SP, Brasil) em dose única de $30 \mathrm{~mL}\left(6 \times 10^{6} \mathrm{UI}\right.$ de vitamina A; $1,2 \times 10^{6} \mathrm{UI}$ de vitamina D3 e $1,8 \times 10^{3}$ UI de vitamina E) por via intramuscular.

Amostras de sangue foram colhidas por venopunção em tubos contendo anticoagulante EDTA $\mathrm{K}_{3}$, para realização do hemograma, e em tubos contendo anticoagulante heparina para avaliação da função leucocitária. Os momentos de colheita avaliados foram aos 20 minutos antes da aplicação das vitaminas (momento zero, M0), três (M1) e dez dias após (M2) a aplicação das vitaminas.

Os números totais de eritrócitos e leucócitos foram mensurados pelo analisador automático de hematologia $\left(\mathrm{ABC} \operatorname{Vet}^{\circledR}{ }^{\circledR}\right.$, marca $\mathrm{ABX}^{\mathrm{TM}}$ Horiba, Montpellier, França), a contagem diferencial de leucócitos foi realizada por esfregaços sanguíneos corados pela técnica de Rosenfeld, e a diferenciação do padrão leucocitário foi determinada ao microscópio óptico.Para verificação da função leucocitária, avaliou-se a capacidade de fagocitose de Staphylococcus aureus e produção de espécies reativas de oxigênio (ERO) pela oxidação da 2',7' diclorodihidrofluoresceína diacetato (DCFH-DA) na molécula fluorescente de diclorofluoresceina (DCF) pelo peróxido de hidrogênio intracelular, com e sem estímulo in vitro exercido pela fagocitose de $S$. aureus, por citometria de fluxo, de acordo com as técnicas utilizadas por Kampen et al. (2004).

As células sanguíneas foram incubadas com $200 \mu \mathrm{L}$ de DCFH-DA $(0,3 \mathrm{mM})$ e/ou com $100 \mu \mathrm{L}$ de $S$. aureus (ATCC 25923) corada com iodeto de propídio (PI; no catálogo P4170, Sigma-Aldrich, St. Louis, MO, USA) a $37^{\circ} \mathrm{C}$ por 30 minutos. Posteriormente as reações 
foram interrompidas pela adição de $2000 \mu \mathrm{L}$ de solução gelada de ácido etilenodiamino tetra-acético (EDTA) (3mM), e em seguida, procedeu-se duas lises hipotônicas das hemácias. As amostras foram ressuspendidas em Phosphate Buffered Saline - Tampão Fosfato Salino (PBS) e lidas no citômetro de fluxo FACSCalibur (Becton Dickinson Immunocytometry Systems, San Diego, CA). 0 aparelho estava ligado ao computador com o software CellQuest (Becton Dickinson Immunocytometry Systems ${ }^{\mathrm{TM}}$ ), sendo analisados 20 mil eventos. Primeiro separou-se as populações celulares de acordo com a dispersão celular em tamanho (FSC) e granulosidade (SSC). Posteriormente, para cada população (granulócitos e céluas mononucleares), avaliou-se a capacidade de fagocitose de SaPI e produção de ERO basal e estimulada pela bactéria S. aureus analisadas em porcentagem e em intensidade média de fluorescência (IMF).

A análise estatística dos dados foi realizada utilizando o software estatístico Instat Graphpad. Para a avaliação das diferenças entre as médias dos resultados obtidos, foram realizados os testes de análise de variância ANOVA One-way (Unstacked) para dados com distribuição normal. Para todos os resultados, diferiram estatisticamente as análises que apresentaram nível de significância menor ou igual a $5 \%(\mathrm{P} \leq 0,05)$, e tendência estatística as que apresentaram nível de significância maior que $5 \%$ e menor ou igual a $10 \%(0,05>\mathrm{P} \leq 0,1)$ (Sampaio 1998).

\section{RESULTADOS}

Os resultados das análises realizadas pela citometria de fluxo do grupo $\mathrm{C}$ e do grupo $\mathrm{S}$ estão apresentados nos Quadros 1 e 2 .

Pode-se observar diferença estatística entre os grupos para as variáveis: porcentagem de granulócitos, porcenta- gem de granulócitos que produziram ERO, porcentagem de células mononucleares e porcentagem de células mononucleares que produziram ERO em todos os momentos. Porém, em cada grupo, o tratamento não influenciou nestas respostas, indicando que estas influências foram individuais e não pelo tratamento. A porcentagem de granulócitos que fagocitaram se manteve constante no grupo $S$ e tendeu a diminuir no grupo $\mathrm{C}$ no $\mathrm{M} 1(\mathrm{P}=0,1)$ apresentando tendência a serem diferentes entre os dois grupos nos $\mathrm{M} 1(\mathrm{P}=0,1)$ e M2 $(\mathrm{P}=0,1)$. Para as células mononucleares, este comportamento foi o oposto, pois notou-se que a porcentagem de células que fagocitaram se mantiveram constante ao longo do tempo no grupo C e diminuíram no grupo S (M0 e M1 diferente de $\mathrm{M} 2 \mathrm{P}=0,01)$, havendo diferença entre os dois grupos desde o $\mathrm{M} 0(\mathrm{M} 0 \mathrm{P}=0,03 ; \mathrm{M} 1 \mathrm{P}=0,004 ; \mathrm{M} 2 \mathrm{P}=0,02)$.

De maneira similar, observou-se que a porcentagem de granulócitos que produziram ERO sob estimulação aumentou no grupo $S$ (M1 diferente M0 e M2 $P=0,0001$ ) e diminuiu no grupo $\mathrm{C}$ (M0 diferente de $\mathrm{M} 1$ e $\mathrm{M} 2 \mathrm{P}=0,0007)$, havendo diferença entre os grupos no M1 $(\mathrm{P}=0,001)$, enquanto que a porcentagem de células mononucleares que produziram ERO sob estimulação se mantiveram constante ao longo do tempo no grupo $\mathrm{C}$ e diminuíram no grupo $\mathrm{S}$ no M1 (M1 diferente de M0 e M2 P=0,0004).

Observou-se diferença entre os grupos, em todos os momentos, para a maioria das variáveis de intensidade média de fluorescência (IMF) estudadas. 0 tratamento promoveu aumento na IMF das atividades dos granulócitos e mono-

Quadro 1. Porcentagem das funções leucocitárias sanguíneas de bezerros suplementados (S) ou não (C). São Paulo, 2015

\begin{tabular}{|c|c|c|c|c|c|c|c|c|}
\hline \multirow{3}{*}{$\begin{array}{l}\text { Funções } \\
\text { Leucocitárias }\end{array}$} & \multirow[t]{3}{*}{ Grupos } & \multicolumn{6}{|c|}{ Momentos } & \multirow[t]{3}{*}{$\mathrm{P}^{\mathrm{a}}$} \\
\hline & & \multicolumn{2}{|c|}{ M0 } & \multicolumn{2}{|c|}{ M1 } & \multicolumn{2}{|c|}{ M2 } & \\
\hline & & Média & EM & Média & EM & Média & EM & \\
\hline \multirow[t]{3}{*}{ \% Granulócitos } & S & 26,35 & 2,15 & 26,04 & 1,59 & 26,74 & 2,11 & 0,95 \\
\hline & $\mathrm{C}$ & 33,12 & 8,11 & 39,35 & 10,90 & 28,31 & 10,11 & 0,13 \\
\hline & $\mathrm{P}^{\mathrm{b}}$ & \multicolumn{2}{|c|}{0,02} & \multicolumn{2}{|c|}{0,71} & \multicolumn{2}{|c|}{0,01} & \\
\hline \% Granulócitos & $\mathrm{S}$ & 99,41 & 0,45 & 99,40 & 0,22 & 99,62 & 0,22 & 0,41 \\
\hline \multirow[t]{2}{*}{ ERO } & $\mathrm{C}$ & 98,20 & 0,34 & 96,31 & 4,05 & 98,72 & 1,23 & 0,18 \\
\hline & $\mathrm{P}^{\mathrm{b}}$ & \multicolumn{2}{|c|}{0,002} & \multicolumn{2}{|c|}{0,2} & \multicolumn{2}{|c|}{0,09} & \\
\hline \% Granulócitos & $\mathrm{S}$ & 93,30 & 4,28 & 90,65 & 4,50 & 92,88 & 3,85 & 0,53 \\
\hline \multirow[t]{2}{*}{ SAPI } & $\mathrm{C}$ & 90,51 & 11,90 & 82,48 & 7,31 & 88,87 & 6,01 & 0,1 \\
\hline & $\mathrm{P}^{\mathrm{b}}$ & \multicolumn{2}{|c|}{0,58} & \multicolumn{2}{|c|}{0,1} & \multicolumn{2}{|c|}{0,1} & \\
\hline \% Granulócitos & $\mathrm{S}$ & 78,32 & 10,20 & 91,91 & 11,88 & 79,40 & 8,74 & 0,0001 \\
\hline \multirow[t]{2}{*}{ EROe } & $\mathrm{C}$ & 81,80 & 13,48 & 46,22 & 19,88 & 76,83 & 8,22 & 0,0007 \\
\hline & $\mathrm{P}^{\mathrm{b}}$ & \multicolumn{2}{|c|}{0,66} & \multicolumn{2}{|c|}{0,001} & \multicolumn{2}{|c|}{0,69} & \\
\hline \multirow[t]{3}{*}{ \% Mononucleares } & $\mathrm{S}$ & 60,47 & 8,49 & 60,75 & 5,48 & 53,65 & 9,33 & 0,15 \\
\hline & $\mathrm{C}$ & 50,62 & 11,18 & 49,90 & 7,42 & 54,40 & 12,85 & 0,7 \\
\hline & $\mathrm{P}^{\mathrm{b}}$ & \multicolumn{2}{|c|}{0,07} & \multicolumn{2}{|c|}{0,03} & \multicolumn{2}{|c|}{0,87} & \\
\hline \% Mononuclear & $\mathrm{S}$ & 96,6 & 2,37 & 94,91 & 1,91 & 95,77 & 1,68 & 0,29 \\
\hline \multirow[t]{2}{*}{ ERO } & $\mathrm{C}$ & 79,18 & 10,39 & 83,93 & 6,05 & 79,34 & 6,95 & 0,74 \\
\hline & $\mathrm{P}^{\mathrm{b}}$ & \multicolumn{2}{|c|}{0,005} & \multicolumn{2}{|c|}{0,0008} & \multicolumn{2}{|c|}{0,004} & \\
\hline$\%$ Mononuclear & $\mathrm{S}$ & 79,31 & 5,03 & 73,75 & 4,95 & 68,38 & 8,13 & 0,01 \\
\hline SAPI & $\mathrm{C}$ & 57,7 & 19,19 & 51,06 & 6,62 & 55,46 & 8,25 & 0,61 \\
\hline & $\mathrm{P}^{\mathrm{b}}$ & & 03 & & 04 & & & \\
\hline$\%$ Mononuclear & S & 40,54 & 5,67 & 25,75 & 5,26 & 45,44 & 10,78 & 0,0004 \\
\hline EROe & $\mathrm{C}$ & 28,25 & 10,07 & 45,53 & 19,03 & 38,52 & 11,91 & 0,12 \\
\hline & $\mathrm{P}^{\mathrm{b}}$ & & 03 & & 02 & & & \\
\hline
\end{tabular}

$\overline{\mathrm{EM}}=$ Erro da média, ERRO produção intracelular de espécies reativas de oxigênio, SAPI = fagocitose de Staphylococcus aureus, EROe = produção intracelular de espécies reativas de oxigênio após estimulação, M0 = 20 minutos antes do tratamento, M1 = três dias após e, M2 = dez dias após o tratamento. ${ }^{\mathrm{a}}$ Interação tempo, ${ }^{\mathrm{b}}$ interação tratamento. 
Quadro 2. Intensidade média de fluorescência das funções leucocitárias sanguíneas, expressas em valores arbitrários, de bezerros suplementados (S) ou não (C). São Paulo, 2015

\begin{tabular}{|c|c|c|c|c|c|c|c|c|}
\hline \multirow{3}{*}{$\begin{array}{l}\text { Funções } \\
\text { leucocitárias }\end{array}$} & \multirow[t]{3}{*}{ Grupos } & \multicolumn{6}{|c|}{ Momentos } & \multirow[t]{3}{*}{$\mathrm{P}^{\mathrm{a}}$} \\
\hline & & \multicolumn{2}{|c|}{ M0 } & \multicolumn{2}{|c|}{ M1 } & \multicolumn{2}{|c|}{ M2 } & \\
\hline & & Média & EM & Média & EM & Média & EM & \\
\hline Granulócitos & $\mathrm{S}$ & 1476,0 & 544,8 & 611,8 & 121,2 & 2058,4 & 273,0 & 0,0001 \\
\hline \multirow[t]{2}{*}{ ERO } & $\mathrm{C}$ & 505,0 & 25,4 & 645,8 & 127,6 & 941,8 & 312,7 & 0,12 \\
\hline & $\mathrm{P}^{\mathrm{b}}$ & \multicolumn{2}{|c|}{0,04} & \multicolumn{2}{|c|}{0,69} & \multicolumn{2}{|c|}{0,08} & \\
\hline Granulócitos & $\mathrm{S}$ & 2159,2 & 319,5 & 1133,8 & 265,0 & 3214,5 & 780,9 & 0,0001 \\
\hline \multirow[t]{2}{*}{ SAPI } & $\mathrm{C}$ & 188,4 & 168,9 & 866,8 & 494,15 & 224,7 & 259,6 & 0,01 \\
\hline & $\mathrm{P}^{\mathrm{b}}$ & \multicolumn{2}{|c|}{0,0001} & \multicolumn{2}{|c|}{0,19} & \multicolumn{2}{|c|}{0,0001} & \\
\hline Granulócitos & $\mathrm{S}$ & 6607,8 & 831,3 & 2361,2 & 514,0 & 8423,0 & 559,8 & 0,0001 \\
\hline \multirow[t]{2}{*}{ EROe } & $\mathrm{C}$ & 710,61 & 161,5 & 4365,5 & 3646,8 & 966,7 & 773,4 & 0,06 \\
\hline & $\mathrm{P}^{\mathrm{b}}$ & \multicolumn{2}{|c|}{0,001} & \multicolumn{2}{|c|}{0,17} & \multicolumn{2}{|c|}{0,01} & \\
\hline Mononucleares & $\mathrm{S}$ & 219,6 & 150,5 & 176,5 & 55,1 & 449,2 & 137,6 & 0,002 \\
\hline \multirow[t]{2}{*}{ ERO } & $\mathrm{C}$ & 67,4 & 46,2 & 275,5 & 99,8 & 213,5 & 157,2 & 0,01 \\
\hline & $\mathrm{P}^{\mathrm{b}}$ & \multicolumn{2}{|c|}{0,009} & \multicolumn{2}{|c|}{0,28} & \multicolumn{2}{|c|}{0,01} & \\
\hline Mononucleares & $\mathrm{S}$ & 975,4 & 240,3 & 434,3 & 108,5 & 831,7 & 187,7 & 0,0007 \\
\hline \multirow[t]{2}{*}{ SAPI } & $\mathrm{C}$ & 83,6 & 81,6 & 111,1 & 63,1 & 1354,8 & 216,2 & 0,13 \\
\hline & $\mathrm{P}^{\mathrm{b}}$ & \multicolumn{2}{|c|}{0,0001} & \multicolumn{2}{|c|}{0,001} & \multicolumn{2}{|c|}{0,99} & \\
\hline Mononucleares & $\mathrm{S}$ & 437,0 & 124,7 & 110,8 & 40,2 & 509,8 & 220,3 & 0,0001 \\
\hline \multirow[t]{2}{*}{ EROe } & $\mathrm{C}$ & 765,3 & 979,2 & 103,9 & 782,2 & 70,4 & 115,6 & 0,04 \\
\hline & $\mathrm{Pb}^{\mathrm{b}}$ & \multicolumn{2}{|c|}{0,35} & \multicolumn{2}{|c|}{0,48} & \multicolumn{2}{|c|}{0,0007} & \\
\hline
\end{tabular}

EM = Erro da média, ERRO = produção intracelular de espécies reativas de oxigênio, SAPI = fagocitose de Staphylococcus aureus, EROe = produção intracelular de espécies reativas de oxigênio após estimulação, M0 = 20 minutos antes do tratamento, M1- três dias após e, M2 =

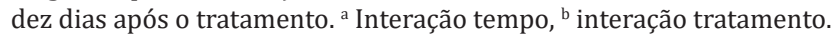

nucleares no M2 em relação ao M1 no grupo S (granulócitos: ERO $\mathrm{P}=0,0001$; fagocitose $\mathrm{P}=0,0001$, ERO estimulado $\mathrm{P}=0,0001$; mononucleares ERO $\mathrm{P}=0,002$, fagocitose $\mathrm{P}=$ 0,0007 e ERO estimulado $\mathrm{P}=0,0001)$. Em relação ao Grupo
C, observou-se aumento na IMF da atividade dos granulócitos no $\mathrm{M} 1$ em relação ao $\mathrm{M} 0$ (fagocitose $\mathrm{P}=0,01$ e ERO estimulada $\mathrm{P}=0,06)$ e da ERO basal das células mononucleares $(\mathrm{P}=0,01)$. No entanto a IMF da ERO estimulada das

Quadro 3. Valores médios dos componentes do eritrograma dos bezerros do grupo suplementado (S) e controle (C). São Paulo, 2015

\begin{tabular}{|c|c|c|c|c|c|c|c|c|}
\hline \multirow[t]{3}{*}{ Constituintes } & \multirow[t]{3}{*}{ Grupos } & \multicolumn{6}{|c|}{ Momentos } & \multirow[t]{3}{*}{$\mathrm{P}^{\mathrm{a}}$} \\
\hline & & \multicolumn{2}{|c|}{ M0 } & \multicolumn{2}{|c|}{ M1 } & \multicolumn{2}{|c|}{ M2 } & \\
\hline & & Média & EM & Média & EM & Média & EM & \\
\hline Hemácia & $\mathrm{S}$ & 7,98 & 0,65 & 8,29 & 0,68 & 8,10 & 0,42 & 0,24 \\
\hline \multirow[t]{2}{*}{$\left(\mathrm{x} 10^{6} / \mathrm{mL}\right)$} & $\mathrm{C}$ & 7,84 & 1,88 & 6,84 & 1,58 & 7,14 & 0,99 & 0,04 \\
\hline & $\mathrm{P}^{\mathrm{b}}$ & \multicolumn{2}{|c|}{0,21} & \multicolumn{2}{|c|}{0,22} & \multicolumn{2}{|c|}{0,37} & \\
\hline Hemoglobina & $S$ & 10,37 & 0,45 & 10,05 & 0,82 & 10,75 & 0,66 & 0,53 \\
\hline \multirow[t]{2}{*}{$(\mathrm{g} / \mathrm{dL})$} & $\mathrm{C}$ & 9,33 & 1,76 & 8,80 & 1,39 & 9,51 & 0,75 & 0,33 \\
\hline & $\mathrm{P}^{\mathrm{b}}$ & \multicolumn{2}{|c|}{0,12} & \multicolumn{2}{|c|}{0,09} & \multicolumn{2}{|c|}{0,01} & \\
\hline Hematócrito & $\mathrm{S}$ & 28,47 & 1,44 & 30,00 & 2,31 & 29,90 & 1,8 & 0,26 \\
\hline \multirow[t]{2}{*}{$(\%)$} & $\mathrm{C}$ & 27,3 & 2,38 & 27,53 & 4,62 & 28,23 & 3,13 & 0,46 \\
\hline & $\mathrm{P}^{\mathrm{b}}$ & \multicolumn{2}{|c|}{0,28} & \multicolumn{2}{|c|}{0,16} & \multicolumn{2}{|c|}{0,15} & \\
\hline \multirow[t]{3}{*}{ VCM (fL) } & $\mathrm{S}$ & 35,87 & 2,90 & 35,33 & 2,80 & 37,08 & 2,99 & 0,42 \\
\hline & $\mathrm{C}$ & 37,55 & 5,30 & 36,82 & 4,20 & 39,65 & 3,80 & 0,13 \\
\hline & $\mathrm{P}^{\mathrm{b}}$ & \multicolumn{2}{|c|}{0,49} & \multicolumn{2}{|c|}{0,44} & \multicolumn{2}{|c|}{0,22} & \\
\hline \multirow{3}{*}{ HCM (pg) } & $\mathrm{S}$ & 13,00 & 0,77 & 12,81 & 0,68 & 13,22 & 0,88 & 0,06 \\
\hline & $\mathrm{C}$ & 12,36 & 1,64 & 11,70 & 1,07 & 13,35 & 1,34 & 0,03 \\
\hline & $\mathrm{P}^{\mathrm{b}}$ & \multicolumn{2}{|c|}{0,40} & \multicolumn{2}{|c|}{0,06} & \multicolumn{2}{|c|}{0,85} & \\
\hline \multirow[t]{3}{*}{ CHCM (g/dL) } & $\mathrm{S}$ & 36,41 & 1,02 & 35,50 & 1,01 & 35,91 & 1,20 & 0,08 \\
\hline & $\mathrm{C}$ & 33,98 & 1,09 & 32,01 & 1,00 & 33,65 & 1,10 & 0,01 \\
\hline & $\mathrm{P}^{\mathrm{b}}$ & \multicolumn{2}{|c|}{0,02} & \multicolumn{2}{|c|}{0,0001} & \multicolumn{2}{|c|}{0,005} & \\
\hline RDW (\%) & $S$ & 19,51 & 2,21 & 19,20 & 2,21 & 18,94 & 2,14 & 0,88 \\
\hline & $\mathrm{C}$ & 17,06 & 1,70 & 18,90 & 0,14 & 17,92 & 2,47 & 0,63 \\
\hline & $\mathrm{P}^{\mathrm{b}}$ & 0 & 50 & & & 0 , & & \\
\hline Plaquetas & $\mathrm{S}$ & 403,50 & 109,60 & 461,20 & 112,90 & 430,80 & 115,60 & 0,63 \\
\hline$\left(\mathrm{x} 10^{3} / \mathrm{mm}^{3}\right)$ & $\mathrm{C}$ & 415,14 & 151,30 & 464,14 & 86,53 & 473,28 & 108,90 & 0,62 \\
\hline & $\mathrm{P}^{\mathrm{b}}$ & 0,87 & & 0 & & 0 & & \\
\hline
\end{tabular}


Quadro 4. Valores médios dos componentes do leucograma, expressos em valores absolutos, dos bezerros do grupo suplementado (S) e controle (C). São Paulo, 2015

\begin{tabular}{|c|c|c|c|c|c|c|c|c|}
\hline \multirow[t]{3}{*}{ Constituintes } & \multirow[t]{3}{*}{ Grupos } & \multicolumn{6}{|c|}{ Momentos } & \multirow[t]{3}{*}{$\mathrm{P}^{\mathrm{a}}$} \\
\hline & & \multicolumn{2}{|c|}{ M0 } & \multicolumn{2}{|c|}{ M1 } & \multicolumn{2}{|c|}{ M2 } & \\
\hline & & Média & EM & Média & EM & Média & EM & \\
\hline Neutrófilos & $\mathrm{S}$ & 1979,5 & 1223,9 & 2318,2 & 1162,9 & 3404,2 & 1053,1 & 0,07 \\
\hline \multirow{2}{*}{$\left(10^{3} / \mu \mathrm{L}\right)$} & $\mathrm{C}$ & 1769,1 & 786,2 & 2201,4 & 814,3 & 2167,7 & 717,1 & 0,51 \\
\hline & $\mathrm{P}^{\mathrm{b}}$ & \multicolumn{2}{|c|}{0,6} & \multicolumn{2}{|c|}{0,8} & \multicolumn{2}{|c|}{0,04} & \\
\hline Linfócitos & $\mathrm{S}$ & 6037,4 & 1822,6 & 6512,5 & 1787,4 & 6446,1 & 1355,9 & 0,84 \\
\hline \multirow{2}{*}{$\left(10^{3} / \mu \mathrm{L}\right)$} & $\mathrm{C}$ & 5238,1 & 1052,1 & 5455,5 & 1423,8 & 5971,4 & 1183,3 & 0,53 \\
\hline & $\mathrm{P}^{\mathrm{b}}$ & \multicolumn{2}{|c|}{0,33} & \multicolumn{2}{|c|}{0,24} & \multicolumn{2}{|c|}{0,49} & \\
\hline Monócitos & $\mathrm{S}$ & 754,0 & 278,4 & 561,7 & 250,4 & 394,0 & 160,5 & 0,03 \\
\hline \multirow[t]{2}{*}{$\left(10^{3} / \mu \mathrm{L}\right)$} & $\mathrm{C}$ & 192,5 & 177,1 & 390,7 & 172,7 & 356,7 & 255,6 & 0,18 \\
\hline & $\mathrm{P}^{\mathrm{b}}$ & \multicolumn{2}{|c|}{0,003} & \multicolumn{2}{|c|}{0,16} & \multicolumn{2}{|c|}{0,4} & \\
\hline Eosinófilos & S & 150,0 & 115,6 & 136,0 & 206,3 & 134,5 & 141,5 & 0,98 \\
\hline \multirow[t]{2}{*}{$\left(10^{3} / \mu \mathrm{L}\right)$} & $\mathrm{C}$ & 44,4 & 42,5 & 23,7 & 40,6 & 28,4 & 57,7 & 0,7 \\
\hline & $\mathrm{P}^{\mathrm{b}}$ & \multicolumn{2}{|c|}{0,04} & \multicolumn{2}{|c|}{0,18} & \multicolumn{2}{|c|}{0,1} & \\
\hline Basófilos & $\mathrm{S}$ & 0 & 0 & 0 & 0 & 0 & 0 & 0 \\
\hline \multirow[t]{2}{*}{$\left(10^{3} / \mu \mathrm{L}\right)$} & $\mathrm{C}$ & 0 & 0 & 0 & 0 & 0 & 0 & 0 \\
\hline & $\mathrm{P}^{\mathrm{b}}$ & \multicolumn{2}{|c|}{0} & \multicolumn{2}{|c|}{0} & \multicolumn{2}{|c|}{0} & \\
\hline Leucócitos & $S$ & 9314,2 & 1831,6 & 9528,5 & 2291,8 & 9985,7 & 2204,1 & 0,83 \\
\hline \multirow{2}{*}{$\left(10^{3} / \mu \mathrm{L}\right)$} & $\mathrm{C}$ & 7642,8 & 1335,2 & 8071,4 & 1387,7 & 8128,5 & 1359,8 & 0,76 \\
\hline & $\mathrm{P}^{\mathrm{b}}$ & \multicolumn{2}{|c|}{0,38} & \multicolumn{2}{|c|}{0,17} & \multicolumn{2}{|c|}{0,24} & \\
\hline
\end{tabular}

$\overline{\mathrm{EM}}=$ Erro da média, $\mathrm{VCM}=$ volume corpuscular médio, $\mathrm{HCM}=$ hemoglobina corpuscular média, $\mathrm{CHCM}=$ concentração de hemoglobina corpuscular média, $\mathrm{M} 0=20$ minutos antes do tratamento, M1 = três dias após e M2 = dez dias após o tratamento. ${ }^{\mathrm{a}}$ Interação tempo, ${ }^{\mathrm{b}}$ interação tratamento.

células mononucleares apresentou redução no M1 e M2 em relação ao $\mathrm{M} 0 \mathrm{P}=0,04)$.

Os resultados das análises realizadas dos hemogramas e leucogramas do grupo $\mathrm{C}$ e do grupo $\mathrm{S}$ estão descritas nos Quadros 3 e 4

Quando observados os componentes do eritrograma, notou-se diminuição dos valores de hemácia e dos índices HCM e CHCM no M1 para o grupo C (hemácia e HCM M1 diferente de M2 $\mathrm{P}=0,04$ e 0,03 respectivamente e CHCM, M1 diferente de M0 e M2 P=0,01), havendo valores menores de hemoglobina (M1 P=0,09, M2 P=0,01), HCM (M1 $\mathrm{P}=0,06)$ e CHCM (M0 $\mathrm{P}=0,02$, M1 $\mathrm{P}=0,0001$ e M2 $\mathrm{P}=0,005$ ) no grupo $\mathrm{C}$ em comparação ao grupo $\mathrm{S}$.

No grupo $S$, encontrou-se tendência a diminuir os índices HCM e CHCM no M1 ( $\mathrm{P}=0,06$ e $\mathrm{P}=0,08$ respectivamente). Para as demais variáveis não houve influência do tratamento.

Em relação aos leucócitos, observou-se diferença estatística entre os grupos no M0 para os monócitos $(\mathrm{P}=0,003)$ e eosinófilos $(\mathrm{P}=0,04)$. Os valores absolutos de eosinófilos se mantiveram constate nos dois grupos independente do tratamento, enquanto que os valores de monócitos diminuíram no grupo $\mathrm{S}$, havendo diferença estatística entre o M0 e M2 $(\mathrm{P}=0,03)$.

Observou-se também tendência a aumentar os valores absolutos de neutrófilos no M2 em relação ao M1 para o Grupo $S(P=0,07)$, sendo estes valores maiores que os do grupo $\mathrm{C}(\mathrm{P}=0,04)$.

\section{DISCUSSÃO}

Acredita-se que a dieta exclusiva de feno causou redução na resposta imune no grupo $\mathrm{C}$ tendo em vista que as porcentagens de granulócitos que fagocitaram e produziram ERO sob estimulação foram menores no M1 relação ao M0. De maneira similar, Graugnard et al. (2012) encontraram redução da atividade imune de neutrófilos de vacas alimentadas com alimento conservado em forma de feno e silagem. Apesar desta redução na porcentagem de células ativas, houve aumento de número de partículas bacterianas fagocitadas e de produção de ERO estimulada por células, indicando possivelmente uma tentativa compensatória para manter a efetividade da resposta imune.

No momento posterior, M2, é possível que os animais já estivessem mais adaptados a dieta, reequilibrando a microbiota ruminal, o que culminou em melhor digestibilidade do alimento, tal qual relatado por Mallmann et al. (2006), ao avaliar a digestibilidade de feno de baixa qualidade por bovinos. Situação semelhante foi observado por Calderón et al. (2007), ao verificarem que bovinos com dieta exclusiva de feno e silagem aumentavam os teores séricos de vitamina $\mathrm{E}$ e beta caroteno após um período de adaptação a dieta. Possivelmente este incremento sérico é o responsável pela melhora das funções dos granulócitos no grupo C.

Em relação às células mononucleares, houve praticamente nenhum efeito da dieta nas funções de fagocitose e produção de ERO, apenas diminuição da IMF de produção de ERO por célula nos M1 e M2.

Para o grupo $S$, notou-se que o suplemento vitamínico melhorou a atividade das células granulocíticas, pois, apesar deles receberem a mesma dieta, foram capazes de manter a porcentagem destas células fagocitando constante nos três momentos avaliados e ainda, apresentaram incremento na porcentagem de granulócitos produzindo ERO estimulada no M1 e aumentaram a IMF destas funções no M2. Utilizando metodologias diferentes, Bouwstra et al. (2008), 
Urban-Chmiel et al. (2009) e Bertagnon et al. (2014) também evidenciaram melhorias na função de fagocitose e produção de ERO estimulada por granulócitos, quando bovinos foram suplementados com vitaminas A e E. Tal efeito pode ser atribuído pela ação destas vitaminas na sinalização intracelular de neutrófilos, resultando em liberação de citocinas que ativam as funções de fagocitose e produção de ERO por neutrófilos (Higuchi \& Nagahata 2000).

Em relação às células mononucleares, encontrou-se diminuição da porcentagem destas células produzindo ERO estimulada no M1 e diminuição da porcentagem de células mononucleares fagocitando no M2. Em contrapartida, as células que estavam exercendo estas atividades, aumentaram sua capacidade, fagocitando e produzindo mais ERO estimulada no M2.

Tendo em vista que neutrófilos são células mais numerosas e mais ativas nos processos de fagocitose e produção de ERO que monócitos (Burton et al. 2005), acredita-se que as alterações das funções dos monócitos foram compensadas ou até mesmo superadas pelo incremento das funções granulocíticas. Possivelmente este é o motivo que os trabalhos similares a esta pesquisa, só avaliaram as funções de neutrófilos e não de monócitos (Bouwstra et al. 2008, Lopes et al. 2009, Urban-Chmiel et al. 2009, Bertagnon et al. 2014).

Para as variáveis do hemograma, observou-se que todos os valores estavam dentro dos parâmetros de normalidade para a espécie (Peixoto et al. 2012). Mesmo assim, os dados do eritrograma reforçam a hipótese de que a dieta exclusiva de feno diminuiu o aporte nutricional para os animais no M1, tendo em vista que neste período encontrou-se diminuição dos valores de hemácia e dos índices HCM e CHCM no grupo C. Estes índices, indicam respectivamente peso e concentração de hemoglobina da hemácia. Sua redução é resultado de falta de substrato para composição da hemoglobina, cuja causa mais comum nos bovinos, é a carência nutricional, especialmente de vitaminas do complexo B (Weiss \& Wardrop 2010). De maneira similar, estes índices também diminuíram no grupo $\mathrm{S}$, porém de maneira menos expressiva e sem redução do número de hemácias, indicando que a suplementação minimizou a carência nutricional. Estes resultados corroboram com os encontrados por Lopes et al. (2009) e Bertagnon et al. (2014) ao estudarem respectivamente caprinos e bovinos suplementados com vitamina E. Estes autores atribuíram este efeito a ação da vitamina na produção dos eritrócitos pela medula óssea e a redução da lipoperoxidação da membrana eritrocitária, mantendo uma maior quantidade de eritrócitos íntegros na circulação periférica.

$\mathrm{O}$ fato da vitamina $\mathrm{E}$ auxiliar na capacidade antioxidante do bovino, reduz as formas reativas de oxigênio intracelular basal (Riegel 2002, Lambeth 2004), tal como observado pelas reduções da intensidade da produção de ERO basal intracelular nos granulócitos e células mononucleares no M1 para os animais do Grupo S (Quadro 2). Tal fato impede o acúmulo destes metabólitos no meio celular, aumentando a viabilidade das hemácias e neutrófilos (Urban-Chmiel et al. 2009), conforme observado no presente trabalho (Quadros 3 e 4).

Ainda, em relação aos leucócitos, observou-se diferen- ça entre os grupos no M0 para os monócitos $(\mathrm{P}=0,003)$ e eosinófilos $(\mathrm{P}=0,04)$, não encontrando explicação para o fato, já que os animais eram de lotes de raça, idade, sexo e peso homogêneos, e criados sob as mesmas condições experimentais. Fatores estes que podem afetar os parâmetros do hemograma (Birgel Junior et al. 2001). É provavel que a redução do aporte nutricional, especialmente de vitaminas do complexo B afetou estas células, porém, por serem em menor número na circulação em comparação a neutrófilos, linfócitos e hemácias (Weiss \& Wardrop 2010), estas diferenças entre os grupos foram evidentes apenas no M0não se repetindo ao longo do experimento.

\section{CONCLUSÕES}

Conclui-se que a suplementação com polivitamínicos A, D e E surtiu efeito benéfico na resposta imune de bezerros da Raça Holandesa, apesar dos efeitos deletérios da alimentação exclusiva com feno e da privação parcial da incidência solar direta, tendo em vista que este tratamento aumentou a atividade granulocítica, diminuiu a porcentagem da atividade das células mononucleares; porém as células que estavam funcionando, apresentaram incremento de sua atividade.

Ainda, observou-se maior efetividade do efeito antioxidante, pelo aumento da sobrevida de hemácias e neutrófilos.

Agradecimentos.- Os autores agradecem o financiamento da bolsa de iniciação científica (FMVZ-USP-PIBIC 2015-3153).

Declaração de conflito de interesse.- Os autores não têm interesses conflitantes.

\section{REFERÊNCIAS}

Baldin S.R., Mille D.D., Martins C.L., Pereira A.S.C., Barducci R.S. \& Arrigoti M.B. 2013. Feedlot performance, carcass characteristics and meat quality of Nellore and Canchim bulls fed diets supplemented with vitamins D and E. Acta Scient. 35(4):403-410.

Bertagnon H.G., Silva E.B., Conneglian M.M., Neumann M., Esper G.V.Z., Bastos G.P. \& Ramos J.P. 2014. Ação imunomoduladora da vitamina E na imunidade sistêmica e da glândula mamária de bovinos leiteiros alimentados com silagem. Semina, Ciênc. Agrárias 35(2):857-866.

Birgel Júnior E.H., D’Angelo J.L., Benesi F.J. \& Birgel E.H. 2001. Valores de referências do leucograma de bovinos da raça Jersey criados no Estado de São Paulo. Braz. J. Vet. Res. Anim. Sci. 38:136-141.

Bouwstra, R.J., Goselink R.M.A., Dobbelaar P., Nielen M., Newbold J.R. \& Van Werven T. 2008. The relationship between oxidative damage and vitamin e concentration in blood, milk, and liver tissue from vitamin $\mathrm{E}$ supplemented and non supplemented periparturient heifers. J. Dairy Sci. 91(3):977-987.

Burton J.L., Madsen S.A., Chang L., Weber P.S.D., Buckham K.R., Van Dorp R., Hickey M.C. \& Earley B. 2005. Gene expression signatures in neutrophils exposed to glucocorticoids: A new paradigm to help explain "neutrophil dysfunction" in parturient dairy cows. Vet. Immunol. Immunopatol. 105:197-219.

Calderón F., Chauveau-Duriot B., Pradel P., Martin B., Graulet B., Doreau M. \& Noziere P. 2007. Variations in carotenoids, vitamins A and E, and color in cow's plasma and milk following a shift from hay diet to diets containing increasing levels of carotenoids and vitamin E. J. Dairy Sci. 90:5651-5664.

Carnagey K.M., Huff-Lonergan E.J., Trenkle A., Wertz-Lutz A., Horst R.L. \& Beitz D.C. 2008. Use of 25- hidroxy vitamin D3 and vitamin E to improve tenderness of beef from longissimus dorsi of heifers. J. Anim. Sci. 86(7):1649-1657. 
Ferreira A.M., Costa J.N., Peixoto A.P., Brito O.S., Cassetari M.L. \& Neto A.O. 2007. Suplementação com vitamina $E$ e a ocorrência de mastites em vacas da raça Jersey. Revta Bras. Saúde Prod. Anim. 8(2):71-82.

Graugnard D.E., Bionaz M., Trevisi E., Moyes K.M., Salak-Johnson J.L., Wallace R.L., Drackley J.K., Bertoni G. \& Loor J.J. 2012. Blood immunometabolic indices and polymorphonuclear neutrophil function in peripartum dairy cows are altered by level of dietary energy prepartum. J. Dairy Sci. 95:1749-1758.

Higuchi H. \& Nagata H. 2000. Effects of vitamins A and E on superoxide production and intracellular signaling of neutrophils in Holstein calves. Can. J. Vet. Res. 64:69-75

Kampen A.H., Tollersrud T. \& Lund A. 2004. Flow cytometric measurement of neutrophil respiratory burst in whole bovine blood using live Staphylococcus aureus. J. Immunol. Meth. 289:47-55.

Lambeth J.D. 2004. NOX enzymes and the biology of reative oxygen. Nat. Rev. Immunol. 4(3):181-118.

Lopes S.T.A., Paes P.R.O., Kohayagawa A., Lopes R.S., Langoni H., Bulla C. \& Langrafe L. 2009. Metabolismo oxidativo dos eritrócitos e eritrograma na mastite induzida por Staphylococcus aureus em cabras suplementadas com vitamina E. Ciênc. Anim. Bras. 10(4):1171-1176.

Mallmann G.M., Patino H.O., Silveira A.L.F., Medeiros F.S. \& Knor M. 2006. Consumo e digestibilidade de feno de baixa qualidade suplementado com nitrogênio não protéico em bovinos. Pesq. Agropec. Bras. 41(2): 331-337.

McDowell L.R., Williams S.N., Hidiroglou N., Njeru C.A., Hill G.M., Ochoa L.
\& Wilkinson N.S. 1996. Vitamin E supplementation for the ruminant. Anim. Feed Sci. Tech. 60(3):273-296.

Mukherjee R. 2008. Selenium and vitamin E increases polymorphonuclear cell phagocytosis and antioxidant levels during acute mastitis in riverine buffaloes. Vet. Res. Comm. 32(4):305-313.

Paschoal J.J., Zanetti M.A. \& Cunha J.A. 2003. Suplementação de selênio e vitamina E sobre a contagem de células somáticas no leite de vacas da raça holandesa. Revta Bras. Zootec. 32(6):2032-2039.

Peixoto A.P.C., Costa J.N., Kohayagawa A., Takahira R.K. \& Saito M.E. 2012. Hemograma e metabolismo oxidativo dos neutrófilos de bovinos da raça Holandesa preta e branca: influência dos fatores etários. Revta Bras. Saúde Prod. Anim. 3(1):16-20.

Politis I. 2012. Reevaluation of vitamin E supplementation of dairy cows: bioavailability, animal health and milk quality. Anim. 6(9):1427-1434.

Riegel R.E. 2002. Bioquímica. 3aㅡ ed. Unisino, São Leopoldo, p.507-536.

Sampaio I.B.M. 1998. Estatística Aplicada à Experimentação Animal. Fundação de Ensino e Pesquisa em Medicina Veterinária e Zootecnia, Belo Horizonte. 221p.

Urban-Chmiel R., Kankofer M., Wernicki A., Albera E. \& Puchalski A. 2009. The influence of different doses of $\alpha$-tocopherol and ascorbic acid on selected oxidative stress parameters in in vitro culture of leukocytes isolated from transported calves. Livst. Sci. 124(1/3):89-92.

Weiss D.J. \& Wardrop K.J. 2010. Schalm's Veterinary Hematology. 6th ed. Wiley and Blackwell, Iowa. 